\title{
BMJ Open Antifungal (oral and vaginal) therapy for recurrent vulvovaginal candidiasis: a systematic review protocol
}

\author{
Juliana Lírio, ${ }^{1}$ Paulo Cesar Giraldo, ${ }^{2}$ Rose Luce Amaral, ${ }^{2}$ \\ Ayane Cristine Alves Sarmento, ${ }^{3}$ Ana Paula Ferreira Costa, ${ }^{3}$ \\ Ana Katherine Gonçalves ${ }^{3}$
}

To cite: Lírio J, Giraldo PC, Amaral RL, et al. Antifungal (oral and vaginal) therapy for recurrent vulvovaginal candidiasis: a systematic review protocol. BMJ Open 2019;9:e027489. doi:10.1136/ bmjopen-2018-027489

- Prepublication history for this paper is available online. To view these files, please visit the journal online (http://dx.doi. org/10.1136/bmjopen-2018027489).

Received 24 October 2018 Accepted 11 March 2019
Check for updates

(c) Author(s) (or their employer(s)) 2019. Re-use permitted under CC BY-NC. No commercial re-use. See rights and permissions. Published by BMJ.

${ }^{1}$ Obstetrics and Gynecology, Universidade Estadual de Campinas, Campinas, SP, Brazil ${ }^{2}$ Obstetrics and Gynecology, Universidade Estadual de Campinas, Campinas, Brazil

${ }^{3}$ Universidade Federal do Rio Grande do Norte, Natal, Brazil

Correspondence to Dr Ana Katherine Gonçalves; anakatherine_ufrnet@yahoo. com.br

\section{ABSTRACT}

Introduction Vulvovaginal candidiasis (VVC) is frequent in women worldwide and usually responds rapidly to topical or oral antifungal therapy. However, some women develop recurrent vulvovaginal candidiasis (RVVC), which is arbitrarily defined as four or more episodes every year. RVVC is a debilitating, long-term condition that can severely affect the quality of life of women. Most VVC is diagnosed and treated empirically and women frequently self-treat with over-the-counter medications that could contribute to an increase in the antifungal resistance. The effective treatment of RVVC has been a challenge in daily clinical practice. This review aims to assess the efficacy of antifungal agents administered orally or intravaginally for the treatment of RVVC, in order to define clinical practices that will impact on the reduction of the morbidity and antifungal resistance.

Methods and analysis A comprehensive search of the following databases will be carried out: PubMed, Embase, Scopus, Web of Science, Scientific Electronic Library Online (SciELO), the Cochrane Central Register of Controlled Trials (CENTRAL), Biblioteca Virtual em Saúde (Virtual Health Library)/Biblioteca Regional de Medicina (Regional Library of Medicine) (BVS/BIREME), Cumulative Index to Nursing and Allied Health Literature (CINAHL) and in the clinical trials databases (www.trialscentral.org; www.controlled-trials.com; www.clinicaltrials.gov). The risk of bias will be assessed according to the Cochrane Risk of Bias tool. We will perform data synthesis using the Review Manager (RevMan) software V.5.2.3. To assess heterogeneity, we will compute the 12 statistic.

Ethics and dissemination This study will be a review of published data and it is not necessary to obtain ethical approval. Findings of this systematic review will be published in a peer-reviewed journal.

Trial registration number CRD42018093817

\section{INTRODUCTION}

\section{Description of the condition}

Vulvovaginal candidiasis (VVC) is frequent in women worldwide and usually responds rapidly to topical or oral antifungal therapy. However, some women develop recurrent vulvovaginal candidiasis (RVVC), which is

\section{Strengths and limitations of this study}

- Two independent reviewers will select studies, extract data without different variables and assess the risk of bias, to indicate through evidence-based medicine if there is a more effective antifungal therapeutic regimen for the treatment of recurrent vulvovaginal candidiasis.

- There may be a limitation of outcome from treatment variation, routes of administration, different doses and quality of the randomised trials used in the systematic review.

- This review and meta-analysis will combine the results of various studies that have comparable sizes of an effect that can be computed.

- However, it may be that we have only a small sample size and a limited number of studies, which may influence the validity and reliability of the findings.

arbitrarily defined as at least three symptomatic episodes in the previous 12 months. ${ }^{1-3}$

It is estimated that RVVC affects approximately 138 million women worldwide annually and 492 million over their lifetimes. ${ }^{12}$ Women reported the period of RVVC to be 1-2 years although a substantial number had symptoms for 4 or 5 years and some very much longer, with risk and symptoms lasting decades. $^{45}$

$\mathrm{C}$ albicans is responsible for the majority of infections in women with RVVC; however, adequate treatment of RVVC requires species determination confirmed by laboratory findings and effective treatment. ${ }^{2}$

Several factors have been associated to RVVC such as genetic (polymorphism, familial, ethnicity), immune mechanisms (HIV, uncontrolled diabetes, steroids, antibiotics, hormone replacement therapy), behavioural (oral sex, oral contraceptive, intercourse frequency) and idiopathic. ${ }^{6-10}$

Fluconazole is inexpensive and well-tolerated medication that is easily administered orally and is the most used antifungal drug. 
However, in the last decade, fluconazole resistance has been reported of women with RVVC. Earlier epidemiologic studies found that almost all women diagnosed with fluconazole-resistant $\mathrm{C}$ albicans had experienced previous exposure to fluconazole. ${ }^{11}$ The rates of azole resistance are highly variable, and they may be influenced by the prescribing patterns of clinicians for both the treatment of and prophylaxis.

In addition, it is still important to recognise that the excessive use and overuse of such topical agents have had other adverse consequences such as oedema, irritability of the skin and maybe even chronic vulvar pain condition (vulvodynia)..$^{12} 13$

Furthermore, it is recognised that there are several factors (genetics, polymorphisms, behavioural and host factors) associated with the pathogenesis of RVVC. In this context, it is unlikely to find one regimen fit for all patients. However, no published studies are comparing different antifungal regimens; thus, this review based on evidence must be useful for practitioners and physicians.

\section{Description of the intervention}

Current treatment options for VVC include antifungal agents sold without a prescription for oral or intravaginal use. Fluconazole has been used extensively while having an unknown impact on fungal susceptibility. ${ }^{11}$

The most commonly used regimen for RVVC consists of 10-14 days of induction therapy with a topical antifungal agent or oral fluconazole, $150 \mathrm{mg}$, followed by fluconazole, $150 \mathrm{mg}$ per week for 6 months (strong recommendation with high quality evidence). ${ }^{14}{ }^{15}$ It was seen that women with RVVC with vulvar excoriation, longer disease time and family history of atopic disease are at greater risk of not responding to maintenance treatment with fluconazole. ${ }^{16}$

In the last decade, isolated cases of women with RVVC who have not responded to fluconazole induction therapy have been reported. After excluding lack of adherence to treatment, resistance to fluconazole should be considered. ${ }^{217-19}$

A previous Cochrane review aimed to compare the clinical cure rate of topical versus oral treatment for the treatment of $\mathrm{VVC}^{20}$ and found no difference in the efficacy of oral and vaginal treatment but found that women generally preferred oral treatment. The recommended treatment regimen for RVVC, as described in the clinical guidelines ${ }^{21}$ whether oral or topical, is not effective for all women. ${ }^{17}$ Side effects reported include headache, abdominal pain and nausea with oral treatment ${ }^{22-24}$ and dyspareunia or irritation with vaginal treatment. ${ }^{24}$ In addition, long-term treatments are expensive, and approximately $50 \%$ of women experience recurrence of symptoms a few months after treatment completion. ${ }^{25}$

Effective treatment of RVVC, with adequate control of symptoms and eradication of the fungus, represents a challenge in daily clinical practice. Many antifungal regimens are available for treatment, some of them with adverse effects that end up reducing women's adherence to treatment. The lack of clear criteria for indication of available drugs and their free use due to self-medication by women has contributed to the increasing antifungal resistance found in some clinical trials.

\section{How the intervention might work}

Antifungal agents generally act as fungistatics and most often work by just destroying the cell wall. Nowadays, despite the great diversity of antifungal agents available for vaginal or systemic use and the large number of clinical trials performed, there are actually very few that compare their efficacy along with the risk of developing resistance.

\section{Why it is important to perform this review}

In order to find a rational use of the antifungal medications available for the treatment of RVVC, as well as the choice of the best route of administration, it is necessary to evaluate comparatively the various proposed schemes normally used. In this way, the choice of the best treatment can be made according to the proven and acceptable safety and efficacy dictates.

By avoiding drugs of doubtful or unproven efficacy, as well as high risk/benefitindex, drug combinations of the same formulations or duplicity of drugs for the same clinical indication, the quality of medical care can be improved.

This study also contributes to the assessment of whether there is a more cost-effective and efficient therapeutic approach for the patient and the health system, between two or more equally effective treatments.

If there is similarity of efficacy between different antifungal drugs used in an oral treatment regimen, one can recommend the one that presents less side effects, more dosage convenience or even lower cost.

In cases of vaginal treatments with superior or similar efficacy to those used orally, they may be chosen as the first option, especially for patients with oral side effects.

Since the sale of antifungal drugs is not subject to prescription control by pharmacies, the indiscriminate use of antifungal drugs by self-medication and without medical prescription has contributed to the increase of antifungal resistance to these drugs. Knowing the efficacy profile of each drug in the treatment of RVVC will enable the creation of a treatment protocol for the pathology and also decrease the risk of increased antifungal resistance.

\section{OBJECTIVES}

To evaluate the efficacy of different antifungal drugs usually used orally and vaginally in the treatment of RVVC.

\section{METHODS}

This systematic review study with probable meta-analysis will follow the criteria: Preferred Reporting Items for Systematic Reviews and Meta-Analyses (PRISMA) 
guidelines. This protocol has been registered with the international prospective register of systematic review.

\section{Criteria for considering studies for this review \\ Types of studies \\ Randomised, blind, published and unpublished clinical trials evaluating treatments for RVVC in immunocompe- tent women will be considered for inclusion.}

\section{Types of participants}

Women who will be included in the analysis will have had four or more episodes of vaginal candidiasis confirmed by the presence of symptoms and a culture or symptoms and positive microscopy. Women with diabetes mellitus and pregnant women will be included in the review but analysed separately in subgroups. Women with immunosuppressive conditions or users of immunosuppressive drugs will be excluded.

\section{Types of interventions}

Interventions to be considered will be antifungal treatments: antifungal drugs administered intravaginally (eg, butoconazole, clotrimazole, econazole, fenticonazole, isoconazole, miconazole, omoconazole, oxiconazole, terconazole, tioconazole, natamycin, sertaconazole nystatin and amphotericin) or oral antifungals (eg, fluconazole, ketoconazole, itraconazole, posaconazole and voriconazole)

The following comparisons will be made: any treatment versus placebo; short duration of treatment versus longer duration of treatment; systemic versus local treatment; Ttreatment of partner versus placebo;

comparison of two different classes of drugs; comparison of different doses of the same agent.

\section{Types of outcome measures}

Primary outcomes: number of clinical recurrences per patient per year (recurrence defined as clinical characteristics and positive culture or microscopy); proportion of participants with at least one clinical recurrence during the treatment and follow-up period.

Secondary outcomes: time for first recurrence; number of symptomatic days per year; number of mycological recurrences per patient per year; proportion of participants with at least one recurrence during treatment and follow-up period; duration of symptoms after starting treatment; complications; adverse events; and patient preference.

\section{Search methods for identification of studies Electronic searches}

We will try to identify as many published studies as unpublished ones, which evaluate the interventions that aim to control RVVC. We will use the electronic search in the databases as the manual search. No language restrictions will be used. The list of databases is shown in box 1 .

\section{Box 1 List of databases}

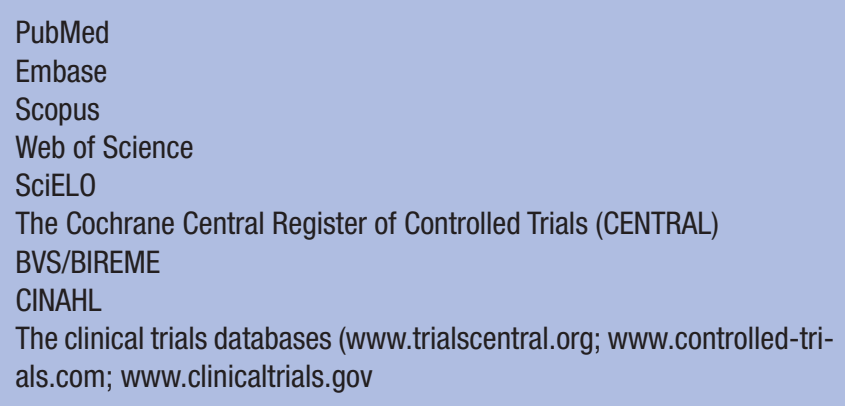

\section{Other sources}

The aim of the computerised bibliographic research will be extended using the reference lists of selected articles.

\section{Search strategy}

The search strategy for Pubmed is shown in table 1 .

\section{Data collection and analysis}

\section{Selection of studies}

Two review authors (JL and AKG) will independently evaluate the eligibility for inclusion of the trials identified by the survey. Disagreements will be resolved by discussion, involving the contribution of a third author (PCG). The trial authors will be contacted if more information is needed before deciding to include. The selection of the study is summarised in a PRISMA flow diagram (figure 1). Thus, papers that met the criteria will be reviewed in full. After the full review, papers not considered having adequate methodological quality according to the grading of recommendation assessment (GRADE) guidelines will be excluded. A list of excluded studies will be provided, with a brief mention of the reason for exclusion.

\section{Data extraction and management}

We will use the Review Manager software (RevMan 2010) to perform statistical analysis. The experimental populations, methods and measurements of results are considered similar and, in the absence of statistical heterogeneity, we will group the data using a fixed effects model. If statistical heterogeneity is present, we will not group or use a random effects model.

The data will be entered into the Review Manager software (RevMan 5.3), which allows the user to enter protocols as well as complete reviews, including text, study characteristics, comparison tables and study data, as well as perform the meta-analysis of the inserted data.

To assess safety and efficacy among the proposed treatments, the dichotomous data will be extracted from each study and inserted into a $2 \times 2$ contingency table, with subsequent individual determination of odds ratio (OR), to obtain a global summary estimate.

Models of fixed effects or random effects will be chosen depending on the absence or presence of heterogeneity between the studies. 


\section{Table 1 Pubmed search strategy}

\begin{tabular}{|c|c|}
\hline See & \\
\hline 1 & candida \\
\hline 2 & candidiasis \\
\hline 3 & candidosis \\
\hline 4 & yeasts \\
\hline 5 & vaginitis \\
\hline 6 & vulvovaginal \\
\hline 7 & OR/1-6 \\
\hline 8 & antifungal \\
\hline 9 & butoconazole \\
\hline 10 & clotrimazole \\
\hline 11 & econazole \\
\hline 12 & fenticonazole \\
\hline 13 & isoconazole \\
\hline 14 & miconazole \\
\hline 15 & omoconazole \\
\hline 16 & oxiconazole \\
\hline 17 & terconazole \\
\hline 18 & tioconazole \\
\hline 19 & natamycin \\
\hline 20 & sertaconazole \\
\hline 21 & amphotericin \\
\hline 22 & fluconazole \\
\hline 23 & ketoconazole \\
\hline 24 & itraconazole \\
\hline 25 & posaconazole \\
\hline 26 & voriconazole \\
\hline 27 & nystatin \\
\hline 28 & OR/8-28 \\
\hline 29 & (randomized controlled Trial) \\
\hline 30 & (blind method) \\
\hline 31 & (clinical trial) \\
\hline 32 & OR/27-30 \\
\hline 33 & 7 AND 28 AND 30 \\
\hline
\end{tabular}

Risk of bias assessment

Two independent reviewers, JL and AKG, will apply the Cochrane Risk of Bias Tool to evaluate the random sequence of generation, allocation concealment, blinding of participants and evaluation of clinical outcomes. We will also evaluate data from incomplete results, selective reporting, financing and potential conflicts of interest associated with individual trials. The risk of bias will be classified using predetermined criteria as follows: low, high or unclear

\section{Measurements of treatment effect}

This will be carried out using the RevMan Analyses statistical package in Review Manager 5.3. We will calculate the OR for dichotomous data and weight mean difference for continuous data with associated $95 \%$ CI.
Unit of analysis issues

For the cure rate of RVVC, the unit of analysis will be defined as 21 and 30 days after the initiation of therapy. For the recurrence rate of RVVC, 3 months and 6 months following the intervention will be considered as shortterm and long-term follow-up, respectively.

\section{Addressing missing data}

We will try to get any missing data by contacting the first author or co-authors of the article via phone, email or post. If we do not receive the necessary information, data will be excluded from our discussion in the Discussion section.

\section{Assessment of heterogeneity}

Statistical heterogeneity among the studies will be assessed by the I2 statistic ( $<25 \%$, without heterogeneity; $25-50 \%$, moderate heterogeneity; and $>50 \%$, strong heterogeneity). When a significant heterogeneity exists between included studies (I2 >50\%), a random effects model will be used for the analysis; otherwise, the fixed effects model will be used. In addition, we will use the Egger funnel chart to evaluate the possible publication bias.

\section{Assessment of reporting biases}

We will use the Egger funnel chart to evaluate the possible publication bias. A linear regression approach will be used to assess the asymmetry of the funnel plot.

\section{Data synthesis}

This will be carried out using the RevMan Analyses statistical package in Review Manager V.5.3. For dichotomous outcomes, we will derive the OR and $95 \%$ CI for each study. Where there is heterogeneity $\left(\mathrm{I}^{2} \geq ; 75 \%\right)$, a random effects model will be used to combine the trials to calculate the relative risk and 95\% CI, using the DerSimonian-Laird algorithm in the meta for package, a meta-analysis package for $\mathrm{R}$.

Other study characteristics and results will be summarised narratively if the meta-analysis cannot be performed for all or some of the included studies.

\section{Sensitivity analyses}

We will conduct sensitivity analyses to explore the robustness of the findings regarding the study quality and sample size. Sensitivity analyses will be shown in a summary table.

\section{Subgroup analyses}

In the subgroup analysis and heterogeneity investigation, we plan to perform the following subgroup analyses:

1. Sexually active versus non-sexually active women.

2. Pregnant versus non-pregnant Women.

3. Women with diabetes mellitus versus non-diabetic women.

4. Intervention duration: short versus long treatment.

5. Route of administration: topical versus systemic.

6. Candida albicans versus non-albicans. 


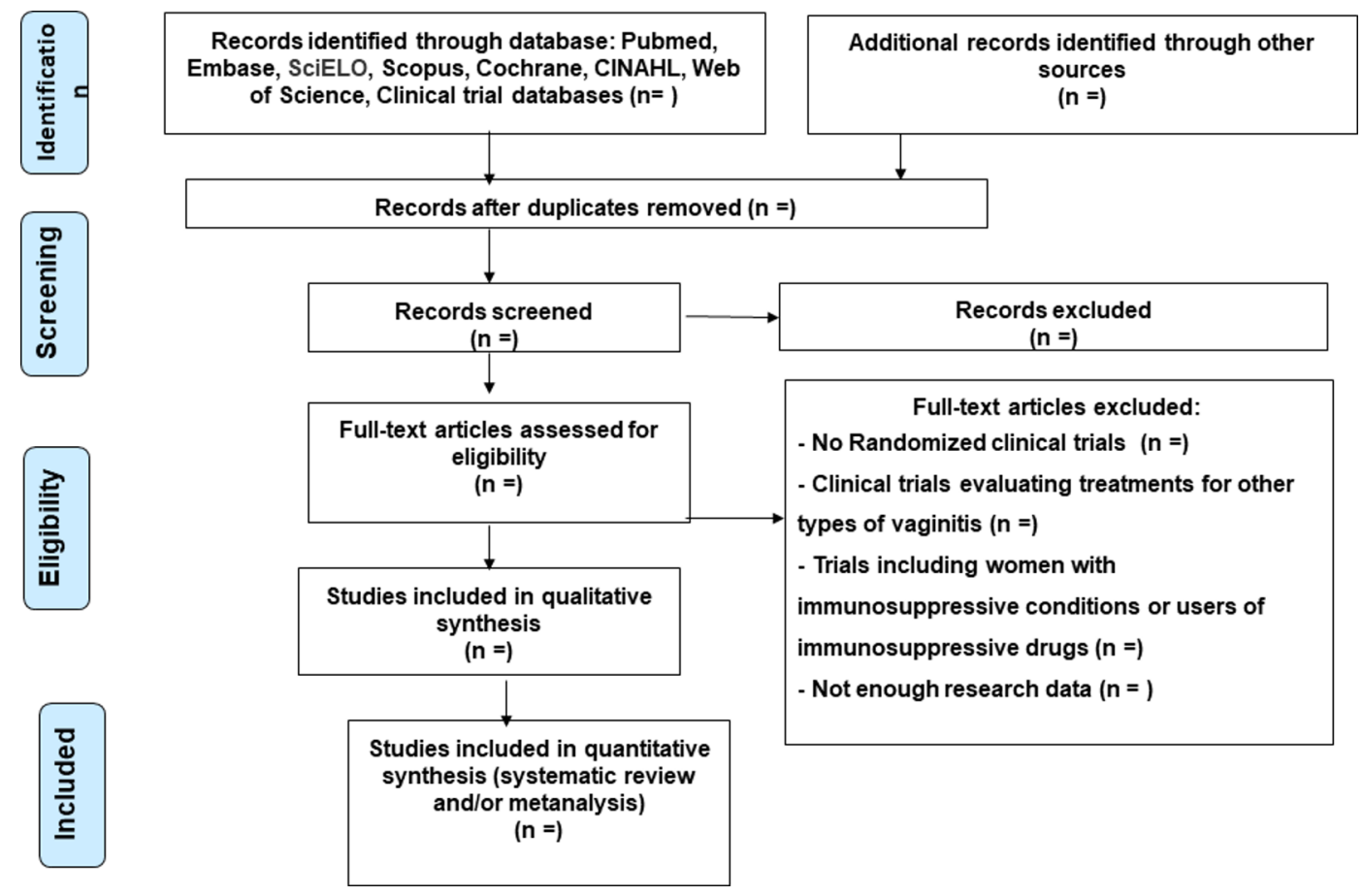

Figure 1 Flow diagram of the search of eligible studies

\section{Confidence in cumulative evidence}

To describe the strength of the evidence for the included data, we will use the GRADE system, which assigns levels of evidence and classifies the strength of the recommendation for health issues. The quality of the evidence will be identified as high if the real effect is close to the estimated effect and moderate if the real impact is probably close to the expected outcome. Additionally, the evidence can be low or very low that is significantly different from the estimated effect. Only, the studies of moderate to a high level of evidence will be included in this review. Patient and public involvement

The research will be performed by a wide and comprehensive search of literature from databases and the individual patient data are not included. Thus, the authors have not involved patients in setting the research question, as well as the outcome measures, the design and implementation of the study, and the dissemination of its results.

\section{DISCUSSION}

RVVC is a prevalent and relevant gynaecological problem, with an impact on women's health. Considerable progress has been made in understanding the pathogenesis of RVVC. It is recognised that diversified factors (genetics, polymorphisms, behavioural, hormonal and host factor) are involved in this process. Thus, it is unlikely to find one regimen fit for all patients. However, no published studies are comparing different antifungal regimens, and in theory, differently in the clinical practice, the different antifungals have similar efficacies, and both routes promote appropriate treatment. In this context, we intend to identify the most effective and safe oral and intravaginal antifungal agents for most women, so this review based on evidence must be useful for practitioners and physicians.

This analysis will provide information on the most effective therapeutic regimens for this prevalent disease, to justify the elaboration of an effective treatment protocol.

\section{Ethics and dissemination}

This study will be a review of the previously published data, so it will not be necessary to obtain approval from the ethics committee. Findings from this systematic review will be published in a peer-reviewed journal.

Acknowledgements The authors acknowledge the assistance provided by the Post-Graduate Program in Obstetrics and Gynecology of UNICAMP in the direction of literary research.

Contributors $\mathrm{JL}$ and AKG contributed to the design of this review. JL drafted the protocol manuscript, and AKG revised it. JL and AKG developed the search strategies and $\mathrm{JL}$ and RLA will implement them. JL and AKG will track potential studies, extract data and assess quality. In case of disagreement between the data extractors, PCG will advise on the methodology and will work as the referee. JL, AKG, RLA, ACAS, APFC and PCG will complete the data synthesis. All authors have approved the final version for publication.

Funding The authors have not declared a specific grant for this research from any funding agency in the public, commercial or not-for-profit sectors.

Competing interests None declared.

Patient consent for publication Not required.

Provenance and peer review Not commissioned; externally peer reviewed.

Open access This is an open access article distributed in accordance with the Creative Commons Attribution Non Commercial (CC BY-NC 4.0) license, which permits others to distribute, remix, adapt, build upon this work non-commercially, and license their derivative works on different terms, provided the original work is properly cited, appropriate credit is given, any changes made indicated, and the use is non-commercial. See: http:// creativecommons.org/licenses/by-nc/4.0/. 


\section{REFERENCES}

1. Denning DW, Kneale M, Sobel JD, et al. Global burden of recurrent vulvovaginal candidiasis: a systematic review. Lancet Infect Dis 2018;18:e339-47.

2. Sobel JD. Recurrent vulvovaginal candidiasis. Am J Obstet Gynecol 2016;214:15-21.

3. Blostein F, Levin-Sparenberg E, Wagner J, et al. Recurrent vulvovaginal candidiasis. Ann Epidemiol 2017;27:575-82.

4. Foxman B, Muraglia R, Dietz JP, et al. Prevalence of recurrent vulvovaginal candidiasis in 5 European countries and the United States: results from an internet panel survey. J Low Genit Tract Dis 2013;17:340-5

5. Ilkit M, Guzel AB. The epidemiology, pathogenesis, and diagnosis of vulvovaginal candidosis: a mycological perspective. Crit Rev Microbiol 2011;37:250-61.

6. Rosentul DC, Delsing CE, Jaeger M, et al. Gene polymorphisms in pattern recognition receptors and susceptibility to idiopathic recurrent vulvovaginal candidiasis. Front Microbiol 2014;5:1-7.

7. Ben-Ali M, Corre B, Manry J, et al. Functional characterization of naturally occurring genetic variants in the human TLR1-2-6 gene family. Hum Mutat 2011;32:643-52.

8. Romani L. Immunity to fungal infections. Nat Rev Immunol 2011;11:275-88.

9. Wojitani MD, de Aguiar LM, Baracat EC, et al. Association between mannose-binding lectin and interleukin-1 receptor antagonist gene polymorphisms and recurrent vulvovaginal candidiasis. Arch Gynecol Obstet 2012;285:149-53.

10. Nedovic B, Posteraro B, Leoncini E, et al. Mannose-binding lectin codon 54 gene polymorphism and vulvovaginal candidiasis: a systematic review and meta-analysis. Biomed Res Int 2014;2014:1-7.

11. Marchaim D, Lemanek L, Bheemreddy S, et al. Fluconazole-resistant Candida albicans vulvovaginitis. Obstet Gynecol 2012;120:1407-14.

12. Leusink $P$, van de Pasch $S$, Teunissen $D$, et al. The Relationship Between Vulvovaginal Candidiasis and Provoked Vulvodynia: A Systematic Review. J Sex Med 2018;15:1310-21.

13. Leusink $P$, van Moorsel $D$, Bor $\mathrm{H}$, et al. Is uncertain vulvovaginal candidiasis a marker of vulvodynia? A study in a Dutch general practice research database. BJGP Open 2017;1:bjgpopen17X100905.
14. Liao X, Qiu H, Li R, et al. Risk factors for fluconazole-resistant invasive candidiasis in intensive care unit patients: An analysis from the China Survey of Candidiasis study. J Crit Care 2015;30:862. e1-e5.

15. Pappas PG, Kauffman CA, Andes DR, et al. Executive Summary: Clinical Practice Guideline for the Management of Candidiasis: 2016 Update by the Infectious Diseases Society of America. Clin Infect Dis 2016;62:409-17.

16. Rosa MI, Silva BR, Pires PS, et al. Weekly fluconazole therapy for recurrent vulvovaginal candidiasis: a systematic review and metaanalysis. Eur J Obstet Gynecol Reprod Biol 2013;167:132-6.

17. Donders GGG, Grinceviciene S, Bellen G, et al. Is non-response to fluconazole maintenance therapy for recurrent Candida vaginitis related to sensitization to atopic reactions? Am J Reprod Immunol 2018;79:e12811.

18. Bulik CC, Sobel JD, Nailor MD. Susceptibility profile of vaginal isolates of Candida albicans prior to and following fluconazole introduction - impact of two decades. Mycoses 2011;54:34-8.

19. Kennedy MA, Sobel JD. Vulvovaginal Candidiasis Caused by Non-albicans Candida Species: New Insights. Curr Infect Dis Rep 2010;12:465-70.

20. Nurbhai M, Grimshaw J, Watson M, et al. Oral versus intra-vaginal imidazole and triazole anti-fungal treatment of uncomplicated vulvovaginal candidiasis (thrush). Cochrane Database Syst Rev 2007:CD002845.

21. Group AE, Spicer JVDUACAGS-CAg. Therapeutic guidelines: antibiotic. 13th edn. North Melbourne, Vic: Therapeutic Guidelines Limited, 2006:442.

22. Pappas PG, Kauffman CA, Andes D, et al. Clinical practice guidelines for the management of candidiasis: 2009 update by the Infectious Diseases Society of America. Clin Infect Dis 2009;48:503-35.

23. Sobel JD, Brooker D, Stein GE, et al. Single oral dose fluconazole compared with conventional clotrimazole topical therapy of Candida vaginitis. Fluconazole Vaginitis Study Group. Am J Obstet Gynecol 1995;172:1263-8.

24. Stein GE, Mummaw N. Placebo-controlled trial of itraconazole for treatment of acute vaginal candidiasis. Antimicrob Agents Chemother 1993;37:89-92.

25. Sobel JD, Wiesenfeld HC, Martens M, et al. Maintenance fluconazole therapy for recurrent vulvovaginal candidiasis. $N$ Engl J Med 2004;351:876-83. 
Correction: Antifungal (oral and vaginal) therapy for

recurrent vulvovaginal candidiasis: a systematic

review protocol

Lírio J, Giraldo PC, Amaral RL, et al. Antifungal (oral and vaginal) therapy for recurrent vulvovaginal candidiasis: a systematic review protocol. BMJ Open 2019;9:e027489. doi: 10.1136/bmjopen-2018-027489.

For this article, an author note has been added below:

It has been noted that this publication covers the same topic as a protocol for a systematic review published by Cochrane in 2011 (https://www.cochranelibrary.com/ cdsr/doi/10.1002/14651858.CD009151/full). The authors acknowledge that they were aware of the protocol and should have cited it in the article. However, the authors have stated that they developed their research question and methods independently.

Open access This is an open access article distributed in accordance with the Creative Commons Attribution Non Commercial (CC BY-NC 4.0) license, which permits others to distribute, remix, adapt, build upon this work non-commercially, and license their derivative works on different terms, provided the original work is properly cited, appropriate credit is given, any changes made indicated, and the use is non-commercial. See: http://creativecommons.org/licenses/by-nc/4.0/.

C Author(s) (or their employer(s)) 2019. Re-use permitted under CC BY-NC. No commercial re-use. See rights and permissions. Published by BMJ.

BMJ Open 2019;9:e027489corr1. doi:10.1136/bmjopen-2018-027489corr1

Check for updates 